\section{DEEP LEARNING REVEALS PREDICTIVE SEQUENCE CONCEPTS WITHIN IMMUNE REPERTOIRES TO IMMUNOTHERAPY}

'John-William Sidhom, 'John-William Sidhom, 'John-William Sidhom*, ${ }^{2}$ Petra RossMacdonald, ${ }^{2}$ Megan Wind-Rotolo, ${ }^{1}$ Andrew Pardoll, ${ }^{1}$ Alexander Baras. 'Johns Hopkins University School of Medicine, Baltimore, MD, USA; ${ }^{2}$ Bristol Myers Squibb, Princeton, NJ, USA

Background Checkpoint inhibition (CPI) has changed the landscape of how oncologists treat advanced cancer ${ }^{1-6}$; while there has been tremendous promise of immunotherapy, most patients do not respond to treatment. ${ }^{6}$ Biomarker development has grown as the field attempts to better select patients that may benefit from immunotherapy as well as further understand effective use of CPI in cancer. ${ }^{7-10}$ One area of interest has been studying the T-cell response through TCR-sequencing, allowing for characterization of the antigenic determinants of response. ${ }^{10-12}$ However, much of the work has been limited to characterizing the quantitative aspects of the TCR repertoire. Here, for the first time, we query whether there are TCR sequence concepts (i.e. motifs) that are predictive of response to immunotherapy.

Methods We employ DeepTCR, ${ }^{13}$ a previously described set of deep learning algorithms, to search for sequence concepts in pre-treatment tumor samples that are predictive of effective immunotherapy in CheckMate 038 (NCT01621490), a clinical trial of CPI. We fit DeepTCR's multiple instance TCR repertoire classifier (figure 1) to predict response (via RECIST) in this cohort and assess not only the predictive performance of the model but insights into an effective antigen-specific T-cell response during CPI.

Results When applying DeepTCR to predict response, a joint representation of TCR repertoire with HLA background of the patient outperformed models that used TCR sequence or HLA genotype information alone (figure 2a). This model's predictions of likelihood to respond to treatment also significantly stratified progression free survival in this cohort of patients (figure 2b). For more qualitative descriptions of the TCR repertoire that defined an effective immune response, we used DeepTCR's variational autoencoder (VAE) to construct an unsupervised representation of the TCR sequences and highlighted the most predictive sequences for responders (blue) and non-responders (red) (figure $3 \mathrm{a}, \mathrm{b}$ ). We noted that not only are the distributions within responders and non-responders multi-modal, but these multiple modes are shared between patients. When comparing the predictive signature in pre- vs post-treatment repertoires, we noted that while the responder signature remained constant over the course of treatment, the non-responder signature demonstrated changes in the TCR sequence space (figure $3 \mathrm{c}, \mathrm{d}$ ).

\section{Supervised TCR Repertoire Classification}
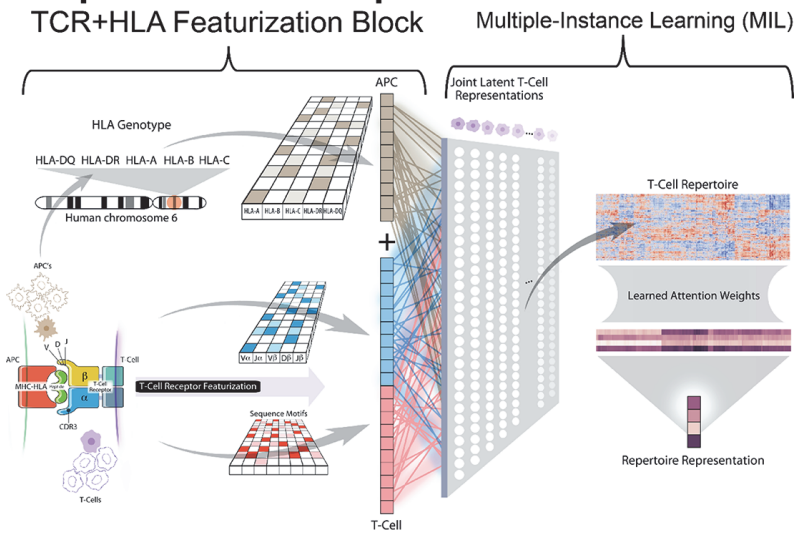

Abstract 832 Figure 1 DeepTCR's multiple instance learning repertoire classifier

We expand on previous work by modifying the DeepTCR Featurization block to incorporate the HLA background within which a given collection of TCRs were observed within. The HLA background of a sample/individual is provided to the neural network in a multi-hot representation that is re-represented in a learned continuous embedding layer and concatenated to the continuous learned representation of the TCR. As previously described, we implement a multi-head attention mechanism to make sequence assignments to concepts within the sample. The number of concepts in the model is a hyperparameter, which can be varied by the user depending on the heterogeneity expected in the repertoires. Of note, this assignment of a sequence to a concept is done through an adaptive activation function that outputs a value between 0 and 1 , allowing the network to put attention on the sequences that are relevant to the learning task. When taking the average of these assignments over all the cells in a repertoire, this results in a value within the neural network that directly corresponds to the proportion of the repertoire that is described by that learned concept. These proportions of concepts in the repertoire are then sent into a final traditional classification layer.

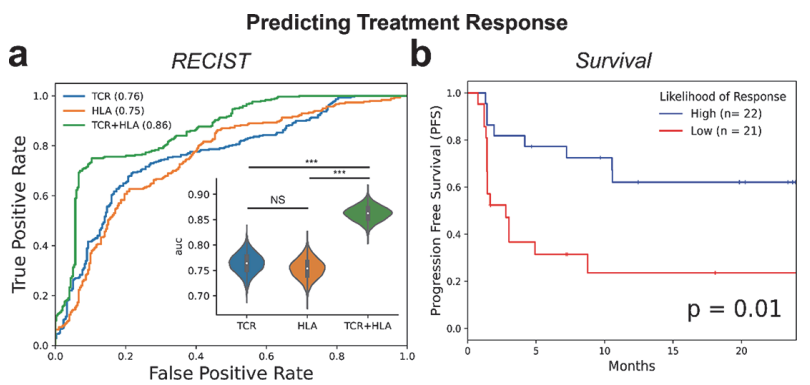

Abstract 832 Figure 2 Repertoire classification in pre-treatment TIL. (A) Pre-treatment tumor biopsies were collected and TCR-Seq was performed from 43 patients enrolled in the CheckMate-038 (parts 2-4) clinical trial where they were either treated with anti-PD1 monotherapy (9 patients) or anti-PD1+ anti-CTLA combination therapy (34 patients) and followed for radiographic response to therapy via RECIST v1.1. Complete Responders and Partial Responders (CRPR) were denoted as responders to therapy while Stable Disease and Progressive Disease (SDPD) were denoted as non-responders to therapy. Receiver Operating Characteristics (ROC) Curves were created for predicting response (complete response, partial response) to immunotherapy given either $\mathrm{TCR}$, HLA, or TCR+HLA information to the supervised repertoire classifier (100 Monte-Carlo simulations with train size: 37 , test size: 6). Bootstrap analyses (5000 iterations) were performed to construct 
confidence intervals $(\mathrm{Cl})$ around AUC values and assess differences in model performance, in which each AUC per sampling was compared in a paired manner across the three models designed above. The null hypothesis of two models exhibiting equivalent performance was rejected if the bootstrap difference did not cross $0 .\left({ }^{* * *}: 99.91 \% \mathrm{Cl}\right)$. (B) The likelihood of response generated by the TCR+HLA model was dichotomized into "High" and "Low" using the median predicted value in this cohort (taken over the MC test sets and averaged per sample) and the Kaplan-Meier (KM) curves were shown for progression free survival (PFS), log-rank $p$-value $=0.005$.

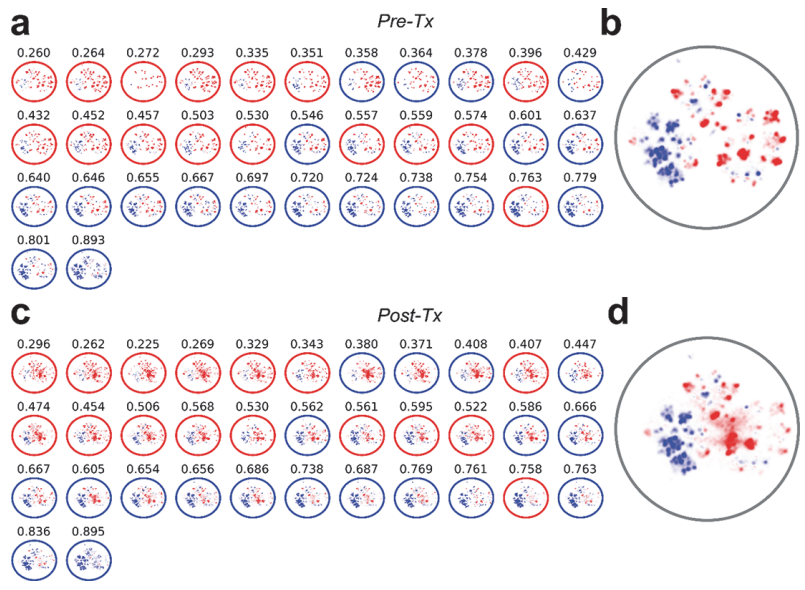

Abstract 832 Figure 3 Pre vs post-treatment TCR repertoire

In order to provide a descriptive understanding of the $\mathrm{T}$ cell response in responders and non-responders in the CheckMate-038 clinical trial, we sought to characterize the distribution of the TCR repertoire in this cohort of patients. Data from CheckMate-038 were used to train a VAE on all sequence data (incorporating TCR + HLA information) in a sample and class agnostic fashion. The distribution of responders and non-responders repertoires were visualized via UMAP of the unsupervised VAE featurization. In order to visualize the distribution of the highly predictive TCR sequences, a per-sequence prediction value was assessed following each MC simulation on the TCR's within the independent test set, assigning the probability that a given TCR had a responder signature. Over the $100 \mathrm{MC}$ simulations, each sequence in the cohort is assigned multiple prediction values that are averaged over all simulations to serve as a consensus predicted value for each sequence in this cohort of patients. Top $10 \%$ of sequences in responders and non-responders were selected and visualized over the entire cohort and on a per-sample basis where edge color denotes the ground truth label of the sample (non-responder $=$ red, responder $=$ blue) and average predicted likelihood taken over MC simulations to respond to treatment shown above each patient's distribution. For each pair of pre/post treatment repertoires, the repertoire-level prediction was compared for pre vs. post treatment across all trained models and the top $10 \%$ of predictive sequences in the 35 paired pre/post repertoires were visualized across all paired samples (a \& c) as well as over the entire cohort (b \& d). (blue $=$ most predictive of response, red $=$ least predictive of response)

Conclusions Taken together, these findings highlight the utility of deep learning to identify sequence features of TCR repertoire under the influence of immunotherapy and note that the pre-existing antigenic response is a key predictor of response to treatment and the maintenance of this antigenic response is a hallmark of clinical benefit.

\section{REFERENCES}

1. Sharma P, Allison JP. (2015). The future of immune checkpoint therapy. Science 348(6230):56-61.

2. Topalian SL, Drake CG, Pardoll DM. (2015). Immune checkpoint blockade: a common denominator approach to cancer therapy. Cancer cell 27(4):450-461.

3. Pardoll DM. (2012). The blockade of immune checkpoints in cancer immunotherapy. Nature Reviews Cancer 12(4):252-264.

4. Hodi FS, O'Day SJ, McDermott DF, Weber RW, Sosman JA, Haanen JB, ... Urba WJ. (2010). Improved survival with ipilimumab in patients with metastatic melanoma. New England Journal of Medicine 363(8):711-723.

5. Robert C, Thomas L, Bondarenko I, O'Day S, Weber J, Garbe C, ... Wolchok JD. (2011). Ipilimumab plus dacarbazine for previously untreated metastatic melanoma. New England Journal of Medicine, 364(26):2517-2526.

6. Topalian SL, Hodi FS, Brahmer JR, Gettinger SN, Smith DC, McDermott DF, ... Sznol M. (2012). Safety, activity, and immune correlates of anti-PD-1 antibody in cancer. New England Journal of Medicine 366(26):2443-2454.

7. Snyder A, Makarov V, Merghoub T, Yuan J, Zaretsky JM, Desrichard A, ... Chan TA. (2014). Genetic basis for clinical response to CTLA-4 blockade in melanoma. New England Journal of Medicine 371(23):2189-2199.

8. Rizvi NA, Hellmann MD, Snyder A, Kvistborg P, Makarov V, Havel JJ, ... Chan TA. (2015). Mutational landscape determines sensitivity to PD-1 blockade in nonsmall cell lung cancer. Science 348(6230):124-128.

9. Van Allen EM, Miao D, Schilling B, Shukla SA, Blank C, Zimmer L, ... Garraway LA. (2015). Genomic correlates of response to CTLA-4 blockade in metastatic melanoma. Science 350(6257):207-211.

10. Sidhom JW, Bessell CA, Havel JJ, Kosmides A, Chan TA, Schneck JP. (2018) ImmunoMap: a bioinformatics tool for T-cell repertoire analysis. Cancer immunology research 6(2):151-162.

11. Riaz N, Havel JJ, Makarov V, Desrichard A, Urba WJ, Sims JS, ... Chan TA (2017). Tumor and microenvironment evolution during immunotherapy with nivolumab. Cell 171(4):934-949.

12. Anagnostou V, Bruhm DC, Niknafs N, White JR, Shao XM, Sidhom JW, ... Velculescu V E. (2020). Integrative tumor and immune cell multi-omic analyses predict response to immune checkpoint blockade in melanoma. Cell Reports Medicine 1 (8):100139.

13. Sidhom JW, Larman HB, Pardoll DM, Baras AS. (2021). DeepTCR is a deep learning framework for revealing sequence concepts within T-cell repertoires. Nature communications 12(1):1-12.

Ethics Approval CheckMate 038 (NCT01621490) is a BMSsponsored, multi-center, institutional-review-board-approved, phase 1 biomarker study of nivolumab, ipilimumab, and nivolumab in combination with ipilimumab in patients with advanced melanoma.

http://dx.doi.org/10.1136/jitc-2021-SITC2021.832 